\title{
Differential Subordination Results for Holomorphic Functions Associated with Wanas Operator
}

\author{
Abbas Kareem Wanas ${ }^{1}$ and Şahsene Altınkaya ${ }^{2}$ \\ ${ }^{1}$ Department of Mathematics, College of Science, University of Al-Qadisiyah, Diwaniyah, Iraq \\ e-mail: abbas.kareem.w@qu.edu.iq \\ ${ }^{2}$ Department of Mathematics, Faculty of Arts and Science, Uludag University, Bursa, Turkey \\ e-mail: sahsene@uludag.edu.tr
}

\begin{abstract}
In this investigation, we define a certain class of holomorphic functions defined by Wanas operator in the open unit disk $U$. We establish some important geometric properties for this class such as inclusion relationship, integral representation and argument estimate.
\end{abstract}

\section{Introduction}

Let $\mathcal{A}$ indicate the family of functions $f$ which are holomorphic in the open unit disk $U=\{z \in \mathbb{C}:|z|<1\}$ and have the following form:

$$
f(z)=z+\sum_{n=2}^{\infty} a_{n} z^{n}
$$

For $\alpha \in \mathbb{R}, \beta \geq 0$ with $\alpha+\beta>0, m, \lambda \in \mathbb{N}_{0}=\mathbb{N} \cup\{0\}$ and $f \in \mathcal{A}$, we consider the differential operator $W_{\alpha, \beta}^{k, \lambda}: \mathcal{A} \rightarrow \mathcal{A}$, introduced by Wanas [11], where

Received: November 27, 2019; Accepted: February 18, 2020

2010 Mathematics Subject Classification: 30C45.

Keywords and phrases: holomorphic function, subordination, integral representation, Wanas operator.

Copyright (C) 2020 Abbas Kareem Wanas and Şahsene Altınkaya. This is an open access article distributed under the Creative Commons Attribution License, which permits unrestricted use, distribution, and reproduction in any medium, provided the original work is properly cited. 


$$
W_{\alpha, \beta}^{k, \lambda} f(z)=z+\sum_{n=2}^{\infty}\left[\sum_{m=1}^{k}\left(\begin{array}{l}
k \\
m
\end{array}\right)(-1)^{m+1}\left(\frac{\alpha^{m}+n \beta^{m}}{\alpha^{m}+\beta^{m}}\right)\right]^{\lambda} a_{n} z^{n}
$$

It is easily verified from (1.2) that

$$
\begin{aligned}
z\left(W_{\alpha, \beta}^{k, \lambda} f(z)\right)^{\prime}= & {\left[\sum_{m=1}^{k}\left(\begin{array}{c}
k \\
m
\end{array}\right)(-1)^{m+1}\left(\left(\frac{\alpha}{\beta}\right)^{m}+1\right)\right] W_{\alpha, \beta}^{k, \lambda+1} f(z) } \\
& -\left[\sum_{m=1}^{k}\left(\begin{array}{l}
k \\
m
\end{array}\right)(-1)^{m+1}\left(\frac{\alpha}{\beta}\right)^{m}\right] W_{\alpha, \beta}^{k, \lambda} f(z) .
\end{aligned}
$$

Some of the special cases of the operator defined by (1.2) can be found in $[1,2,3,8$, $10]$.

Let $T$ stand for the family of mapping $h$ of the form:

$$
h(z)=1+\sum_{n=1}^{\infty} h_{n} z^{n}
$$

which are holomorphic and convex univalent in $U$ and satisfy the condition:

$$
\operatorname{Re}\{h(z)\}>0, \quad(z \in U) .
$$

Given two functions $f$ and $g$ which are holomorphic in $U$, we say that $f$ is subordinate to $g$, written $f \prec g$ or $f(z) \prec g(z)(z \in U)$, if there exists a Schwarz function $w$ which is holomorphic in $U$ with $w(0)=0$ and $|w(z)|<1$ such that $f(z)=g(w(z)),(z \in U)$. In particular, if the function $g$ is univalent in $U$, then we have the following equivalent (see [7]), $f \prec g \Leftrightarrow f(0)=g(0)$ and $f(U) \subset g(U)$.

To establish our main results, we require the following lemmas.

Lemma 1.1 [5]. Let $u, v \in \mathbb{C}$ and suppose that $\psi$ is convex and univalent in $U$ with $\psi(0)=1$ and $\operatorname{Re}\{u \psi(z)+v\}>0,(z \in U)$. If $q$ is holomorphic in $U$ with $q(0)=1$, then the subordination

$$
q(z)+\frac{z q^{\prime}(z)}{u q(z)+v} \prec \psi(z)
$$

implies that $q(z) \prec \psi(z)$. 
Lemma 1.2 [6]. Let $h$ be convex univalent in $U$ and $\mathcal{T}$ be holomorphic in $U$ with $\operatorname{Re}\{\mathcal{T}(z)\} \geq 0,(z \in U)$. If $q$ is holomorphic in $U$ and $q(0)=h(0)$, then the subordination

$$
q(z)+\mathcal{T}(z) z q^{\prime}(z) \prec h(z)
$$

implies that $q(z) \prec h(z)$.

Lemma 1.3 [4]. Let $q$ be holomorphic in $U$ with $q(0)=1$ and $q(z) \neq 0$ for all $z \in U$. If there exists two points $z_{1}, z_{2} \in U$ such that

$$
-\frac{\pi}{2} b_{1}=\arg \left(q\left(z_{1}\right)\right)<\arg (q(z))<\arg \left(q\left(z_{2}\right)\right)=\frac{\pi}{2} b_{2},
$$

for some $b_{1}$ and $b_{2}\left(b_{1}>0, b_{2}>0\right)$ and for all $z\left(|z|<\left|z_{1}\right|=\left|z_{2}\right|\right)$, then

$$
\frac{z_{1} q^{\prime}\left(z_{1}\right)}{q\left(z_{1}\right)}=-i\left(\frac{b_{1}+b_{2}}{2}\right) \eta \text { and } \frac{z_{2} q^{\prime}\left(z_{2}\right)}{q\left(z_{2}\right)}=i\left(\frac{b_{1}+b_{2}}{2}\right) \eta
$$

where

$$
\eta \geq \frac{1-|\varepsilon|}{1+|\varepsilon|} \text { and } \varepsilon=i \tan \frac{\pi}{4}\left(\frac{b_{2}-b_{1}}{b_{1}+b_{2}}\right)
$$

\section{Main Results}

We begin this section by defining the function class $\mathcal{W}(\delta, \alpha, \beta, k, \lambda ; h)$ as follows:

Definition 2.1. A function $f \in \mathcal{A}$ is called in the family $\mathcal{W}(\delta, \alpha, \beta, k, \lambda ; h)$ if it satisfies the following differential subordination:

$$
\frac{1}{1-\delta}\left(\frac{z\left(W_{\alpha, \beta}^{k, \lambda} f(z)\right)^{\prime}}{W_{\alpha, \beta}^{k, \lambda} f(z)}-\delta\right) \prec h(z),
$$

where $\alpha \in \mathbb{R}, \beta \geq 0$ with $\alpha+\beta>0, m, \lambda \in \mathbb{N}_{0}=\mathbb{N} \cup\{0\}, 0 \leq \delta<1$ and $h \in T$.

In the following theorem, we establish the inclusion relationship for the class $\mathcal{W}(\delta, \alpha, \beta, k, \lambda ; h)$. 
Theorem 2.1. Let $\operatorname{Re}\left\{\delta+(1-\delta) h(z)+\sum_{m=1}^{k}\left(\begin{array}{c}k \\ m\end{array}\right)(-1)^{m+1}\left(\frac{\alpha}{\beta}\right)^{m}\right\}>0$. Then

$$
\mathcal{W}(\delta, \alpha, \beta, k, \lambda+1 ; h) \subset \mathcal{W}(\delta, \alpha, \beta, k, \lambda ; h) .
$$

Proof. Assume that $f \in \mathcal{W}(\delta, \alpha, \beta, k, \lambda+1 ; h)$ and put

$$
q(z)=\frac{1}{1-\delta}\left(\frac{z\left(W_{\alpha, \beta}^{k, \lambda} f(z)\right)^{\prime}}{W_{\alpha, \beta}^{k, \lambda} f(z)}-\delta\right)
$$

Then $q$ is holomorphic in $U$ with $q(0)=1$. Making use of the identity (1.3), we find from (2.2) that

$$
\begin{aligned}
& {\left[\sum_{m=1}^{k}\left(\begin{array}{c}
k \\
m
\end{array}\right)(-1)^{m+1}\left(\left(\frac{\alpha}{\beta}\right)^{m}+1\right)\right] \frac{W_{\alpha, \beta}^{k, \lambda+1} f(z)}{W_{\alpha, \beta}^{k, \lambda} f(z)} } \\
= & \delta+(1-\delta) q(z)+\sum_{m=1}^{k}\left(\begin{array}{l}
k \\
m
\end{array}\right)(-1)^{m+1}\left(\frac{\alpha}{\beta}\right)^{m} .
\end{aligned}
$$

Differentiating both sides of (2.3) with respect to $z$ and multiplying by $z$, we have

$$
\begin{aligned}
q(z)+\frac{z q^{\prime}(z)}{\delta+(1-\delta) q(z)+\sum_{m=1}^{k}\left(\begin{array}{c}
k \\
m
\end{array}\right)(-1)^{m+1}\left(\frac{\alpha}{\beta}\right)^{m}} \\
=\frac{1}{1-\delta}\left(\frac{z\left(W_{\alpha, \beta}^{k, \lambda+1} f(z)\right)^{\prime}}{W_{\alpha, \beta}^{k, \lambda+1} f(z)}-\delta\right) \prec h(z) .
\end{aligned}
$$

Since $\operatorname{Re}\left\{\delta+(1-\delta) h(z)+\sum_{m=1}^{k}\left(\begin{array}{l}k \\ m\end{array}\right)(-1)^{m+1}\left(\frac{\alpha}{\beta}\right)^{m}\right\}>0$, applying Lemma 1.1 to the subordination (2.4), yields $q(z) \prec h(z)$, which implies $f \in \mathcal{W}(\delta, \alpha, \beta, k, \lambda ; h)$.

Next, we find integral representation of the class $\mathcal{W}(\delta, \alpha, \beta, k, \lambda ; h)$.

Theorem 2.2. Let $f \in \mathcal{W}(\delta, \alpha, \beta, k, \lambda ; h)$. Then 


$$
W_{\alpha, \beta}^{k, \lambda} f(z)=z \cdot \exp \left[(1-\delta) \int_{0}^{z} \frac{h(w(s))-1}{s} d s\right],
$$

where $w$ is holomorphic in $U$ with $w(0)=0$ and $|w(z)|<1,(z \in U)$.

Proof. Suppose that $f \in \mathcal{W}(\delta, \alpha, \beta, k, \lambda ; h)$. It is easy to see that subordination condition (2.1) can be written as follows

$$
\frac{z\left(W_{\alpha, \beta}^{k, \lambda} f(z)\right)^{\prime}}{W_{\alpha, \beta}^{k, \lambda} f(z)}=(1-\delta) h(w(z))+\delta,
$$

where $w$ is holomorphic in $U$ with $w(0)=0$ and $|w(z)|<1,(z \in U)$.

From (2.5), we find that

$$
\frac{\left(W_{\alpha, \beta}^{k, \lambda} f(z)\right)^{\prime}}{W_{\alpha, \beta}^{k, \lambda} f(z)}-\frac{1}{z}=(1-\delta) \frac{h(w(z))-1}{z} .
$$

After integrating both sides of (2.6), we have

$$
\log \left(\frac{W_{\alpha, \beta}^{k, \lambda} f(z)}{z}\right)=(1-\delta) \int_{0}^{z} \frac{h(w(s))-1}{s} d s .
$$

Therefore, from (2.7), we obtain the required result.

Theorem 2.3. Let $f \in \mathcal{A}, 0<a_{1}, a_{2} \leq 1$ and $0 \leq \delta<1$. If

$$
-\frac{\pi}{2} a_{1}<\arg \left(\frac{z\left(W_{\alpha, \beta}^{k, \lambda+1} f(z)\right)^{\prime}}{W_{\alpha, \beta}^{k, \lambda+1} g(z)}-\delta\right)<\frac{\pi}{2} a_{2},
$$

for some $g \in \mathcal{W}\left(\delta, \alpha, \beta, k, \lambda+1 ; \frac{1+A Z}{1+B z}\right),(-1 \leq B<A \leq 1)$, then

$$
-\frac{\pi}{2} b_{1}<\arg \left(\frac{z\left(W_{\alpha, \beta}^{k, \lambda} f(z)\right)^{\prime}}{W_{\alpha, \beta}^{k, \lambda} g(z)}-\delta\right)<\frac{\pi}{2} b_{2},
$$

where $b_{1}$ and $b_{2}\left(0<b_{1}, b_{2} \leq 1\right)$ are the solutions of the equations: 


$$
a_{1}=\left\{\begin{array}{l}
b_{1}+\frac{2}{\pi} \tan ^{-1}\left(\frac{(1-|\varepsilon|)\left(b_{1}+b_{2}\right) \cos \frac{\pi}{2} t}{2(1+|\varepsilon|)\left(\delta+\frac{(1+A)(1-\delta)}{1+B}+\sum_{m=1}^{k}\left(\begin{array}{l}
k \\
m
\end{array}\right)(-1)^{m+1}\left(\frac{\alpha}{\beta}\right)^{m}\right)}\right. \\
+(1-|\varepsilon|)\left(b_{1}+b_{2}\right) \sin \frac{\pi}{2} t
\end{array}\right), \quad B \neq-1,
$$

and

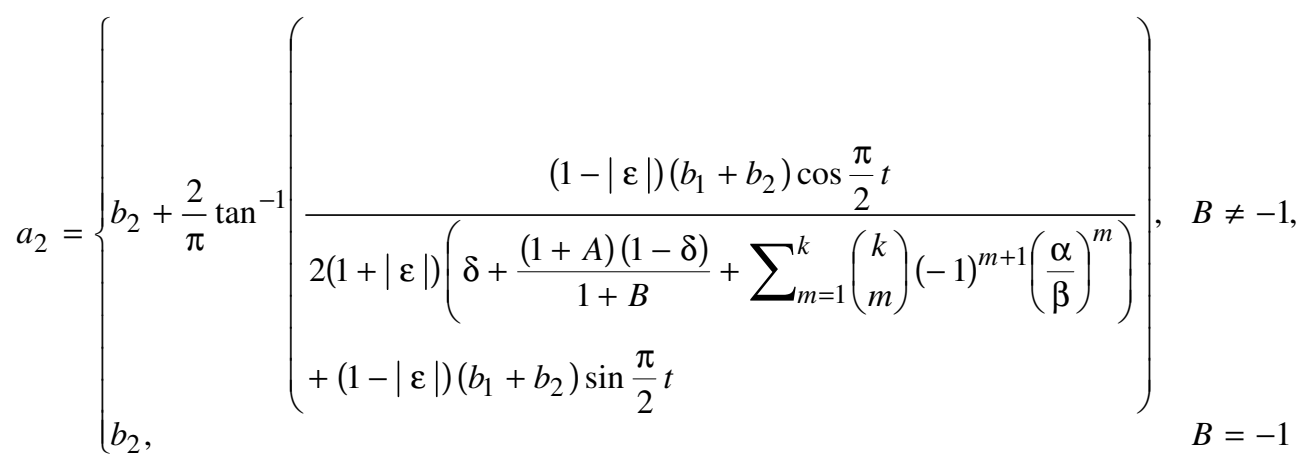

with

$$
\varepsilon=i \tan \frac{\pi}{2}\left(\frac{b_{2}-b_{1}}{b_{1}+b_{2}}\right)
$$

and

$$
t=\frac{2}{\pi} \sin ^{-1}\left(\frac{(A-B)(1-\delta)}{\left(\delta+\sum_{m=1}^{k}\left(\begin{array}{c}
k \\
m
\end{array}\right)(-1)^{m+1}\left(\frac{\alpha}{\beta}\right)^{m}\right)+\left(1-B^{2}\right)+(1-\delta)(1-A B)}\right) .
$$

Proof. Define the function $G$ by 


$$
G(z)=\frac{1}{1-\tau}\left(\frac{z\left(W_{\alpha, \beta}^{k, \lambda} f(z)\right)^{\prime}}{W_{\alpha, \beta}^{k, \lambda} g(z)}-\tau\right),
$$

where $g \in \mathcal{W}\left(\delta, \alpha, \beta, k, \lambda+1 ; \frac{1+A Z}{1+B z}\right),(-1 \leq B<A \leq 1)$ and $0 \leq \tau<1$.

Then $G$ is holomorphic in $U$ with $G(0)=1$. Thus in view of (1.3) and (2.11), we observe that

$$
\begin{aligned}
((1-\tau) G(z)+\tau) W_{\alpha, \beta}^{k, \lambda} g(z)= & {\left[\sum_{m=1}^{k}\left(\begin{array}{c}
k \\
m
\end{array}\right)(-1)^{m+1}\left(\left(\frac{\alpha}{\beta}\right)^{m}+1\right) W_{\alpha, \beta}^{k, \lambda+1} f(z)\right.} \\
& -\left[\sum_{m=1}^{k}\left(\begin{array}{l}
k \\
m
\end{array}\right)(-1)^{m+1}\left(\frac{\alpha}{\beta}\right)^{m}\right] W_{\alpha, \beta}^{k, \lambda} f(z) .
\end{aligned}
$$

Differentiating above relation with respect to $z$ and multiplying by $z$, we get

$$
\begin{aligned}
& ((1-\tau) G(z)+\tau) z\left(W_{\alpha, \beta}^{k, \lambda} g(z)\right)^{\prime}+(1-\tau) z G^{\prime}(z) W_{\alpha, \beta}^{k, \lambda} g(z) \\
= & {\left[\sum_{m=1}^{k}\left(\begin{array}{l}
k \\
m
\end{array}\right)(-1)^{m+1}\left(\left(\frac{\alpha}{\beta}\right)^{m}+1\right)\right] z\left(W_{\alpha, \beta}^{k, \lambda+1} f(z)\right)^{\prime} } \\
& -\left[\sum_{m=1}^{k}\left(\begin{array}{l}
k \\
m
\end{array}\right)(-1)^{m+1}\left(\frac{\alpha}{\beta}\right)^{m}\right] z\left(W_{\alpha, \beta}^{k, \lambda} f(z)\right)^{\prime} .
\end{aligned}
$$

Suppose that

$$
H(z)=\frac{1}{1-\delta}\left(\frac{z\left(W_{\alpha, \beta}^{k, \lambda} g(z)\right)^{\prime}}{W_{\alpha, \beta}^{k, \lambda} g(z)}-\delta\right) .
$$

Using (1.3) again, we have

$$
\left[\sum_{m=1}^{k}\left(\begin{array}{l}
k \\
m
\end{array}\right)(-1)^{m+1}\left(\left(\frac{\alpha}{\beta}\right)^{m}+1\right)\right] \frac{W_{\alpha, \beta}^{k, \lambda+1} g(z)}{W_{\alpha, \beta}^{k, \lambda} g(z)}
$$




$$
=\delta+(1-\delta) H(z)+\left[\sum_{m=1}^{k}\left(\begin{array}{l}
k \\
m
\end{array}\right)(-1)^{m+1}\left(\frac{\alpha}{\beta}\right)^{m}\right] .
$$

From (2.12) and (2.13), we easily get

$$
G(z)+\frac{z G^{\prime}(z)}{\delta+(1-\delta) H(z)+\sum_{m=1}^{k}\left(\begin{array}{c}
k \\
m
\end{array}\right)(-1)^{m+1}\left(\frac{\alpha}{\beta}\right)^{m}}=\frac{1}{1-\tau}\left(\frac{z\left(W_{\alpha, \beta}^{k, \lambda+1} f(z)\right)^{\prime}}{W_{\alpha, \beta}^{k, \lambda+1} g(z)}-\tau\right) .
$$

Notice that from Theorem 2.1, $g \in \mathcal{W}\left(\delta, \alpha, \beta, k, \lambda+1 ; \frac{1+A Z}{1+B z}\right)$ implies $g \in \mathcal{W}\left(\delta, \alpha, \beta, k, \lambda ; \frac{1+A Z}{1+B z}\right)$. Thus,

$$
H(z) \prec \frac{1+A Z}{1+B z} \quad(-1 \leq B<A \leq 1) .
$$

By applying the result of Silverman and Silvia [9], we have

$$
\left|H(z)-\frac{1-A B}{1-B^{2}}\right|<\frac{A-B}{1-B^{2}} \quad(B \neq-1, z \in U)
$$

and

$$
\operatorname{Re}\{H(z)\}>\frac{1-A}{2} \quad(B=-1, z \in U) .
$$

It follows from (2.15) and (2.16) that

$$
\begin{aligned}
\delta & +(1-\delta) H(z)+\sum_{m=1}^{k}\left(\begin{array}{l}
k \\
m
\end{array}\right)(-1)^{m+1}\left(\frac{\alpha}{\beta}\right)^{m} \\
- & \frac{\left(\delta+\sum_{m=1}^{k}\left(\begin{array}{l}
k \\
m
\end{array}\right)(-1)^{m+1}\left(\frac{\alpha}{\beta}\right)^{m}\right)\left(1-B^{2}\right)+(1-\delta)(1-A B)}{1-B^{2}}
\end{aligned}
$$




$$
<\frac{(A-B)(1-\delta)}{1-B^{2}},(B \neq-1, z \in U)
$$

and

$$
\begin{aligned}
& \operatorname{Re}\left\{\delta+(1-\delta) H(z)+\sum_{m=1}^{k}\left(\begin{array}{l}
k \\
m
\end{array}\right)(-1)^{m+1}\left(\frac{\alpha}{\beta}\right)^{m}\right\} \\
> & \delta+\frac{(1-A)(1-\delta)}{2}+\sum_{m=1}^{k}\left(\begin{array}{l}
k \\
m
\end{array}\right)(-1)^{m+1}\left(\frac{\alpha}{\beta}\right)^{m},(B=-1, z \in U) .
\end{aligned}
$$

Putting

$$
\delta+(1-\delta) H(z)+\sum_{m=1}^{k}\left(\begin{array}{l}
k \\
m
\end{array}\right)(-1)^{m+1}\left(\frac{\alpha}{\beta}\right)^{m}=\rho e^{i \frac{\pi}{2} \phi},
$$

where

$$
\begin{gathered}
-\frac{(A-B)(1-\delta)}{\left(\delta+\sum_{m=1}^{k}\left(\begin{array}{l}
k \\
m
\end{array}\right)(-1)^{m+1}\left(\frac{\alpha}{\beta}\right)^{m}\right)\left(1-B^{2}\right)+(1-\delta)(1-A B)}<\phi \\
<\frac{(A-B)(1-\delta)}{\left(\delta+\sum_{m=1}^{k}\left(\begin{array}{c}
k \\
m
\end{array}\right)(-1)^{m+1}\left(\frac{\alpha}{\beta}\right)^{m}\right)\left(1-B^{2}\right)+(1-\delta)(1-A B)}, \quad(B \neq-1)
\end{gathered}
$$

and $-1<\phi<1,(B=-1)$, then

$$
\begin{gathered}
\delta+\frac{(1-A)(1-\delta)}{1-B}+\sum_{m=1}^{k}\left(\begin{array}{c}
k \\
m
\end{array}\right)(-1)^{m+1}\left(\frac{\alpha}{\beta}\right)^{m}<\rho \\
<\delta+\frac{(1+A)(1-\delta)}{1+B}+\sum_{m=1}^{k}\left(\begin{array}{l}
k \\
m
\end{array}\right)(-1)^{m+1}\left(\frac{\alpha}{\beta}\right)^{m},(B \neq-1)
\end{gathered}
$$

and

$$
\delta+\frac{(1-A)(1-\delta)}{1-B}+\sum_{m=1}^{k}\left(\begin{array}{l}
k \\
m
\end{array}\right)(-1)^{m+1}\left(\frac{\alpha}{\beta}\right)^{m}<\rho<\infty,(B=-1) .
$$


An application of Lemma 1.2 with

$$
\mathcal{T}(z)=\frac{1}{\delta+(1-\delta) H(z)+\sum_{m=1}^{k}\left(\begin{array}{l}
k \\
m
\end{array}\right)(-1)^{m+1}\left(\frac{\alpha}{\beta}\right)^{m}},
$$

yields $G(z) \prec h(z)$.

If there exist two points $z_{1}, z_{2} \in U$ such that

$$
-\frac{\pi}{2} b_{1}=\arg \left(G\left(z_{1}\right)\right)<\arg (G(z))<\arg \left(G\left(z_{2}\right)\right)=\frac{\pi}{2} b_{2},
$$

then by Lemma 1.3, we get

$$
\frac{z_{1} G^{\prime}\left(z_{1}\right)}{G\left(z_{1}\right)}=-\frac{\eta i}{2}\left(b_{1}+b_{2}\right) \text { and } \frac{z_{2} G^{\prime}\left(z_{2}\right)}{G\left(z_{2}\right)}=\frac{\eta i}{2}\left(b_{1}+b_{2}\right) \text {, }
$$

where

$$
\eta \geq \frac{1-|\varepsilon|}{1+|\varepsilon|} \text { and } \varepsilon=i \tan \frac{\pi}{4}\left(\frac{b_{2}-b_{1}}{b_{1}+b_{2}}\right) .
$$

Now, for the case $B \neq-1$, we obtain

$$
\begin{aligned}
& \arg \left(\frac{1}{1-\tau}\left(\frac{z_{1}\left(W_{\alpha, \beta}^{k, \lambda+1} f\left(z_{1}\right)\right)^{\prime}}{W_{\alpha, \beta}^{k, \lambda+1} g\left(z_{1}\right)}-\tau\right)\right) \\
= & \arg \left(G\left(z_{1}\right)+\frac{z_{1} G^{\prime}\left(z_{1}\right)}{\delta+(1-\delta) H\left(z_{1}\right)+\sum_{m=1}^{k}\left(\begin{array}{c}
k \\
m
\end{array}\right)(-1)^{m+1}\left(\frac{\alpha}{\beta}\right)^{m}}\right) \\
= & \arg \left(G\left(z_{1}\right)\right)+\arg \left(1+\frac{z_{1} G^{\prime}\left(z_{1}\right)}{\left[\delta+(1-\delta) H\left(z_{1}\right)+\sum_{m=1}^{k}\left(\begin{array}{c}
k \\
m
\end{array}\right)(-1)^{m+1}\left(\frac{\alpha}{\beta}\right)^{m}\right] G\left(z_{1}\right)}\right)
\end{aligned}
$$


$=-\frac{\pi}{2} b_{1}+\arg \left(1-\frac{\eta i}{2 \rho}\left(b_{1}+b_{2}\right) e^{-i \frac{\pi}{2} \phi}\right)$

$=-\frac{\pi}{2} b_{1}+\arg \left(1-\frac{\eta}{2 \rho}\left(b_{1}+b_{2}\right) \cos \frac{\pi}{2}(1-\phi)+\frac{\eta i}{2 \rho}\left(b_{1}+b_{2}\right) \sin \frac{\pi}{2}(1-\phi)\right)$

$\leq-\frac{\pi}{2} b_{1}-\tan ^{-1}\left(\frac{\eta\left(b_{1}+b_{2}\right) \sin \frac{\pi}{2}(1-\phi)}{2 \rho+\eta\left(b_{1}+b_{2}\right) \cos \frac{\pi}{2}(1-\phi)}\right)$

$\leq-\frac{\pi}{2} b_{1}-\tan ^{-1}\left(\begin{array}{l}\frac{(1-|\varepsilon|)\left(b_{1}+b_{2}\right) \cos \frac{\pi}{2} t}{2(1+|\varepsilon|)\left(\delta+\frac{(1+A)(1-\delta)}{1+B}+\sum_{m=1}^{k}\left(\begin{array}{c}k \\ m\end{array}\right)(-1)^{m+1}\left(\frac{\alpha}{\beta}\right)^{m}\right)} \\ +(1-|\varepsilon|)\left(b_{1}+b_{2}\right) \sin \frac{\pi}{2} t\end{array}\right)$

$=-\frac{\pi}{2} a_{1}$,

where $a_{1}$ and $t$ are given by (2.8) and (2.10), respectively.

Also,

$$
\arg \left(\frac{1}{1-\tau}\left(\frac{z_{2}\left(W_{\alpha, \beta}^{k, \lambda+1} f\left(z_{2}\right)\right)^{\prime}}{W_{\alpha, \beta}^{k, \lambda+1} g\left(z_{2}\right)}-\tau\right)\right)
$$

$\geq \frac{\pi}{2} b_{2}+\tan ^{-1}\left(\begin{array}{l}\frac{(1-|\varepsilon|)\left(b_{1}+b_{2}\right) \cos \frac{\pi}{2} t}{2(1+|\varepsilon|)\left(\delta+\frac{(1+A)(1-\delta)}{1+B}+\sum_{m=1}^{k}\left(\begin{array}{c}k \\ m\end{array}\right)(-1)^{m+1}\left(\frac{\alpha}{\beta}\right)^{m}\right)} \\ +(1-|\varepsilon|)\left(b_{1}+b_{2}\right) \sin \frac{\pi}{2} t\end{array}\right)$ 
$=\frac{\pi}{2} a_{2}$,

where $a_{2}$ and $t$ are given by (2.9) and (2.10), respectively.

Similarly, for the case $B=-1$, we have

$$
\arg \left(\frac{1}{1-\tau}\left(\frac{z_{1}\left(W_{\alpha, \beta}^{k, \lambda+1} f\left(z_{1}\right)\right)^{\prime}}{W_{\alpha, \beta}^{k, \lambda+1} g\left(z_{1}\right)}-\tau\right)\right) \leq-\frac{\pi}{2} b_{1}
$$

and

$$
\arg \left(\frac{1}{1-\tau}\left(\frac{z_{2}\left(W_{\alpha, \beta}^{k, \lambda+1} f\left(z_{2}\right)\right)^{\prime}}{W_{\alpha, \beta}^{k, \lambda+1} g\left(z_{2}\right)}-\tau\right)\right) \geq \frac{\pi}{2} b_{2} .
$$

The above two cases disagree with the assumptions. Therefore, the proof of the theorem is complete.

\section{References}

[1] F. M. Al-Oboudi, On univalent functions defined by a generalized Sălăgean operator, Int. J. Math. Math. Sci. 2004, Article ID 172525, 8 pp. https://doi.org/10.1155/S0161171204108090

[2] N. E. Cho and T. H. Kim, Multiplier transformations and strongly close-to-convex functions, Bull. Korean Math. Soc. 40(3) (2003), 399-410. https://doi.org/10.4134/BKMS.2003.40.3.399

[3] N. E. Cho and H. M. Srivastava, Argument estimates of certain analytic functions defined by a class of multiplier transformations, Math. Comput. Modelling 37(1-2) (2003), 39-49. https://doi.org/10.1016/S0895-7177(03)80004-3

[4] A. Ebadian, S. Shams, Z. G. Wang and Y. Sun, A class of multivalent analytic functions involving the generalized Jung-Kim-Srivastava operator, Acta Univ. Apulensis 18 (2009), 265-277.

[5] P. Eenigenburg, P. T. Mocanu, S. S. Miller and M. O. Reade, On a Briot-Bouquet differential subordination, General Inequalities, Birkhäuser, Basel 3 (1983), 339-348. https://doi.org/10.1007/978-3-0348-6290-5_26 
[6] S. S. Miller and P. T. Mocanu, Differential subordinations and univalent functions, Michigan Math. J. 28 (1981), 157-171. https://doi.org/10.1307/mmj/1029002507

[7] S. S. Miller and P. T. Mocanu, Differential Subordinations: Theory and Applications, Series on Monographs and Textbooks in Pure and Applied Mathematics, Vol. 225, Marcel Dekker Inc., New York and Basel, 2000.

[8] G. S. Salagean, Subclasses of univalent functions, Lecture Notes in Math., Springer Verlag, Berlin, 1013 (1983), 362-372. https://doi.org/10.1007/BFb0066543

[9] H. Silverman and E. M. Silvia, Subclasses of starlike functions subordinate to convex functions, Canad. J. Math. 37 (1985), 48-61. https://doi.org/10.4153/CJM-1985-004-7

[10] S. R. Swamy, Inclusion properties of certain subclasses of analytic functions, Int. Math. Forum 7(36) (2012), 1751-1760.

[11] A. K. Wanas, New differential operator for holomorphic functions, Earthline J. Math. Sci. 2(2) (2019), 527-537. https://doi.org/10.34198/ejms.2219.527537 